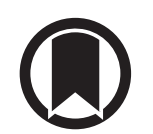

CrossMark

\title{
Gene-based diagnosis of tuberculosis with a new-generation pathogen enrichment technique
}

\author{
To the Editors:
}

While the Xpert MTB/RIF assay (Cepheid Sunnyvale, CA, USA) has greater than 95\% sensitivity for identifying acid-fast bacilli (AFB) smear-positive tuberculosis (TB) cases, for smear-negative pulmonary tuberculosis (PTB) the sensitivity was as low as $60 \%[1,2]$. To address the low sensitivity of current TB diagnostics for AFB smear-negative cases, we developed a simple and label-free pathogen enrichment using homobifunctional imidoesters (HIs) using a microfluidic (SLIM) platform followed by conventional Mycobacterium tuberculosis PCR to extract low amounts of pathogens from clinical samples [3]. In this study, we compared the diagnostic performance of the SLIM assay with the Xpert MTB/RIF for PTB diagnosis in a country with an intermediate TB burden and a low human immunodeficiency virus burden. The SLIM assay was performed by using $1 \mathrm{~mL}$ and $2 \mathrm{~mL}$ aliquots of sputum, respectively.

Adult patients for whom sputum TB test was indicated to confirm presumptive active TB were enrolled prospectively over 13 months at two tertiary referral hospitals. TB diagnosis was confirmed independently by three respiratory infection specialists experienced in TB diagnosis and treatment. The institutional review boards of two hospitals evaluated and approved the medical, scientific and ethical aspects of our study protocol (institutional review boards 2018-0020 and 4-2018-0029), and informed consent was obtained from all participants.

Mycobacterial cultures were grown in liquid and solid culture media, and M. tuberculosis complex was identified with a duplex PCR using Advansure TB/NTM real-time PCR (LG Chemistry, Seoul, South Korea). Culture-positive TB cases were patients with at least one positive $M$. tuberculosis culture. Culture-positive patients were considered smear-positive with at least one positive smear (including faintly positive smears). Culture-negative TB cases were patients with a high probability of clinically active TB with negative mycobacterial culture findings after two or more sputum examinations, showing good clinical and radiographic responses to anti-TB treatment during follow-up. The specialists choosing TB treatment were blinded to SLIM assay results. SLIM assay principles and detailed methods have been described previously [3,4]. Briefly, a mixture of sputum sample and liquefaction solution with HIs reagent were added to the SLIM system to enrich M. tuberculosis and DNA extracted without the need for detergents or bulky instruments.

A total of 152 patients were enrolled between January 2018 and January 2019. The mean \pm SD age was $59.6 \pm 16.0$ years, and 104 patients (68.4\%) were male. Only three (2\%) of the 152 patients had HIV infection. 60 patients were diagnosed with TB, 18 with smear-positive culture-positive TB, 19 with smear-negative culture-positive $\mathrm{TB}$, and 23 with smear-negative culture-negative clinical $\mathrm{TB}$. The remaining 92 patients had diseases other than TB. A comparison of the diagnostic performances of Xpert MTB/RIF and SLIM assays based on clinical diagnosis and stratified by $M$. tuberculosis culture results, are presented in table 1 . In patients, the sensitivity for detecting TB by Xpert MTB/RIF, SLIM $1 \mathrm{~mL}$, and SLIM $2 \mathrm{~mL}$ assays were 37\% (95\% CI 25-50\%), 60\% (95\% CI 47-72\%) and 84\% (95\% CI 71-92\%), respectively. SLIM $1 \mathrm{~mL}$ and SLIM $2 \mathrm{~mL}$ sensitivities were significantly higher than Xpert MTB/RIF ( $\mathrm{p}=0.001$ and $\mathrm{p}<0.001$, respectively). The specificity of Xpert MTB/RIF, SLIM $1 \mathrm{~mL}$, and SLIM $2 \mathrm{~mL}$ were 100\% (95\% CI 96-100\%), 91\% (95\% CI 84-96\%) and 87\% (95\% CI 76-94\%), respectively. The sensitivity

@ERSpublications

A new simple pathogen enrichment system, the SLIM assay, is sensitive for AFB smear-negative cases compared to the Xpert MTB/RIF assay for the rapid detection of pulmonary tuberculosis http://bit.ly/ 37lUHTh

Cite this article as: Lee SW, Kang YA, Jin CE, et al. Gene-based diagnosis of tuberculosis with a new-generation pathogen enrichment technique. Eur Respir J 2020; 55: 1901885 [https://doi.org/10.1183/ 13993003.01885-2019]. 
TABLE 1 Diagnostic performance of Xpert MTB/RIF and SLIM assay in 152 patients with presumptive pulmonary tuberculosis (TB)

\begin{tabular}{|c|c|c|c|c|c|}
\hline $\begin{array}{l}\text { Sensitivity \% } \\
\text { (n/N, 95\% CI) }\end{array}$ & $\begin{array}{l}\text { Specificity \% } \\
\text { (n/N, 95\% Cl) }\end{array}$ & $\begin{array}{c}\text { PPV \% } \\
\text { (n/N, } 95 \% \mathrm{CI} \text { ) }\end{array}$ & $\begin{array}{c}\text { NPV \% } \\
\text { (n/N, } 95 \% \mathrm{Cl})\end{array}$ & $\begin{array}{l}\text { Positive likelihood } \\
\text { ratio }(95 \% \mathrm{CI})\end{array}$ & $\begin{array}{l}\text { Negative likelihood } \\
\text { ratio }(95 \% \mathrm{CI})\end{array}$ \\
\hline
\end{tabular}

\begin{tabular}{|c|c|c|c|c|c|c|}
\hline \multicolumn{7}{|c|}{ TB $(n=60)$ compared with not TB $(n=92)$} \\
\hline Xpert MTB/RIF & $37(22 / 60,25-50)$ & $100(92 / 92,96-100)$ & $100(22 / 22,85-100)$ & $71(92 / 130,62-78)$ & Not applicable & $0.63(0.52-0.77)$ \\
\hline SLIM $1 \mathrm{~mL}$ & $60(36 / 60,47-72)$ & $91(84 / 92,84-96)$ & $82(36 / 44,67-92)$ & $78(84 / 108,69-85)$ & $6.90(3.45-13.80)$ & $0.43(0.31-0.60)$ \\
\hline SLIM $2 \mathrm{~mL}^{\#}$ & $84(46 / 55,71-92)$ & $87(54 / 62,76-94)$ & $85(46 / 54,73-93)$ & $86(54 / 63,75-93)$ & $6.48(3.36-12.51)$ & $0.19(0.10-0.34)$ \\
\hline \multicolumn{7}{|c|}{ Culture-positive TB ( $n=37)$ compared with not TB (n=92) } \\
\hline Xpert MTB/RIF & $57(21 / 37,39-73)$ & $100(92 / 92,96-100)$ & $100(21 / 21,84-100)$ & $85(92 / 108,77-91)$ & Not applicable & $0.43(0.29-0.63)$ \\
\hline SLIM $1 \mathrm{~mL}$ & $70(26 / 37,53-84)$ & $91(84 / 92,84-96)$ & $76(26 / 34,59-89)$ & $88(84 / 95,80-94)$ & $8.08(4.03-16.18)$ & $0.33(0.19-0.54)$ \\
\hline SLIM $2 \mathrm{~mL}$ & $91(39 / 43,78-97)$ & $87(54 / 62,76-94)$ & $83(39 / 47,69-92)$ & $93(54 / 58,83-98)$ & $7.03(3.66-13.52)$ & $0.11(0.04-0.27)$ \\
\hline \multicolumn{7}{|c|}{ Culture-negative TB ( $n=23)$ compared with not TB ( $n=92)$} \\
\hline Xpert MTB/RIF & $4(1 / 23,1-22)$ & $100(92 / 92,96-100)$ & $100(1 / 1,3-100)$ & $81(92 / 114,72-87)$ & Not applicable & $0.96(0.88-1.04)$ \\
\hline SLIM $1 \mathrm{~mL}$ & $43(10 / 23,23-65)$ & $91(84 / 92,84-96)$ & $56(10 / 18,31-78)$ & $87(84 / 97,78-93)$ & $5.00(2.23-11.24)$ & $0.62(0.43-0.89)$ \\
\hline SLIM $2 \mathrm{~mL}$ & $76(16 / 21,53-92)$ & $87(54 / 62,76-94)$ & $67(16 / 24,45-84)$ & $92(54 / 59,81-97)$ & $5.90(2.96-11.77)$ & $0.27(0.12-0.59)$ \\
\hline
\end{tabular}

PPV: positive predicted value; NPV: negative predicted value. " : of 152 patients, only $117(77 \%)$ underwent SLIM $2 \mathrm{~mL}$ assay because of limited sample volumes.

of each assay was analysed further based on TB categories: smear-positive, smear-negative culture-positive, and culture-negative clinical TB. In smear-positive TB, sensitivity was $83 \%$ with Xpert MTB/RIF, $94 \%$ with SLIM $1 \mathrm{~mL}, 100 \%$ with SLIM $2 \mathrm{~mL}$. In smear-negative culture-positive TB, the sensitivity was 32\% with Xpert MTB/RIF, 47\% with SLIM $1 \mathrm{~mL}$, and 78\% with SLIM $2 \mathrm{~mL}$; the sensitivity of SLIM $2 \mathrm{~mL}$ was significantly higher than that of Xpert MTB/RIF ( $p=0.02$ ). For culture-negative clinical TB, the sensitivity of Xpert MTB/RIF was only $4 \%$, and the sensitivity of both SLIM assays was significantly higher, $43 \%$ with SLIM $1 \mathrm{~mL}(\mathrm{p}=0.004)$, and 76\% with SLIM $2 \mathrm{~mL}(\mathrm{p}<0.001)$.

In most conventional assays, only a small quantity of clinical sample is used for pathogen detection uses, then the remaining sample unused. However, the SLIM assay uses larger sample volumes (up to $50 \mathrm{~mL}$ ) with simultaneous pathogen concentration and extraction in a single system $[3,4]$, with comparable time to Xpert MTB/RIF (2.5-4.5 h for SLIM) but with significantly lower cost (\$5-6 for SLIM) and instrument requirements for sample processing. In the current study, we showed superior sensitivity of the SLIM assay compared to the Xpert MTB/RIF, especially for detecting smear-negative PTB. However, as pathogen concentration using HIs can bind negatively charged pathogens non-specifically, some false-positives occur. Hence, the SLIM assay showed slightly lower specificity than Xpert MTB/RIF, although the difference was not statistically significant. Notably, the presence of $M$. tuberculosis DNA was also confirmed by Sanger sequencing, and greater than $98 \%$ sequences were identical to $M$. tuberculosis in all positive SLIM assay samples (data not shown). The Xpert MTB/RIF Ultra assay, a new, highly sensitive assay, also had limited specificity in recently treated patients with TB [5]. False-positives may occur because these methods may detect $M$. tuberculosis DNA from accumulated bacilli debris in patients with prior TB history. Another possibility is the rapid elimination of TB pathogen though an innate or acquired immune response after initial detection with transient inflammation following contact [6]. Therefore, we believe that the SLIM assay might be useful for subclinical or incipient TB diagnosis, a recently proposed disease spectrum between latent TB infection and active TB [7]. Further, development of semiquantitative or quantitative PCR results based on the SLIM assay would offer means to interpret test results in patients with culture-negative TB or subclinical TB, since an earlier study showed greater than half of Xpert MTB/ RIF Ultra false-positive results in patients with a history of TB were semiquantitative "trace" results of Xpert MTB/RIF Ultra [5].

Some may argue that about one-third of patients were diagnosed as having clinical PTB based on clinical features and chest computed tomography findings compatible with PTB and their clinical response to anti-tuberculosis therapy, without microbiological confirmation. We included culture-negative TB cases because positive bacterial confirmation alone does not offer adequate sensitivity for evaluating new diagnostic tests, though it is more sensitive than mycobacterial culture [8]. Although we used strictly predefined diagnostic criteria for culture-negative $\mathrm{TB}$ cases in our previous studies $[9,10]$, the use of clinical TB diagnosis is prone to misclassification bias. Therefore, a cautious interpretation is needed for our findings showing sensitivities of SLIM $1 \mathrm{~mL}$ and $2 \mathrm{~mL}$ in culture-negative TB cases of $43 \%$ and $76 \%$, respectively. In addition, it is difficult to draw conclusions based on this small cohort. However, when culture-confirmed PTB cases were included with PTB, the sensitivity of the SLIM assay was substantially higher compared to Xpert MTB/RIF (table 1), and further studies to assess the accuracy of the SLIM assay 
are warranted. While the specificity of the SLIM assay was relatively acceptable (approximately 90\%), the potential for false-positive results must be considered. The use of the new Xpert MTB/RIF Ultra as reference standards or the comparison of SLIM assay to Xpert MTB/RIF Ultra may provide useful data; however, such data was not available during the study period.

In conclusion, the SLIM assay, a gene-based diagnosis method with a new generation concentration technique, improved PTB diagnostic sensitivity compared to the Xpert MTB/RIF. This improvement was more evident in smear-negative $\mathrm{PTB}$, for which new sensitive tests are needed desperately. Further investigation of the SLIM assay to evaluate its utility in detecting paucibacillary TB and further refinement of the assay to reduce false-positives are necessary. Finally, we are developing a fully integrated system that combines the SLIM assay and the optical sensor-based nucleic acid detection techniques for rapid $(<2 \mathrm{~h})$ and highly sensitive diagnosis of pathogens such as TB. The fully integrated system would become useful as a point-of-care test for a variety of samples such as sputum, cerebrospinal fluid, plasma, bronchoalveolar lavage fluid and urine for clinical applications.

Sei Won Lee, ${ }^{1,6}$, Young Ae Kang ${ }^{2,6}$, Choong Eun Jin ${ }^{3,6}$, Ho Cheol Kim ${ }^{1}$, Geun Su Noh ${ }^{3}$, Hyo Joo Lee, Joung Ha Park ${ }^{4}$, Yong Seo Koo ${ }^{5}$, Yong Shin ${ }^{3,7}$ and Sung-Han Kim ${ }^{4,7}$

${ }^{1}$ Dept of Pulmonary and Critical Care Medicine, Asan Mediclal Center, University of Ulsan College of Medicine, Seoul, Republic of Korea. ${ }^{2}$ Division of Pulmonology, Dept of Internal Medicine, Yonsei University College of Medicine, Severance hospital, Seoul, Republic of Korea. ${ }^{3}$ Dept of Convergence Medicine, Asan Medical Center, Asan Medical Institute of Convergence Science and Technology (AMIST), University of Ulsan College of Medicine, Seoul, Republic of Korea. ${ }^{4}$ Dept of Infectious Diseases, Asan Medical Center, University of Ulsan College of Medicine, Seoul, Republic of Korea. ${ }^{5}$ Dept of Neurology, Asan Medical Center, Seoul, Republic of Korea. ${ }^{6}$ These authors contributed equally to this study as first authors. ${ }^{7}$ These authors contributed equally to this study.

Correspondence: Sung-Han Kim, Dept of Infectious Diseases, Asan Medical Center, University of Ulsan College of Medicine, 88, Olympic-ro 43-gil, Songpa-gu, Seoul, 138-736 05505, Republic of Korea. E-mail: kimsunghanmd@hotmail.com

Received: 23 Sept 2019 | Accepted after revision: 14 Nov 2019

Conflict of interest: None declared.

Support statement: This study was supported by grants from Asan Institute for Life Sciences (2019-7040 and 2019-7043) and the Korea Health Technology R\&D Project through the Korea Health Industry Development Institute (KHIDI), funded by the Ministry of Health and Welfare, Republic of Korea (HI16C-0272-010016). Funding information for this article has been deposited with the Crossref Funder Registry.

\section{References}

1 Lee HS, Kee SJ, Shin JH, et al. Xpert MTB/RIF assay as a substitute for smear microscopy in an intermediate-burden setting. Am J Respir Crit Care Med 2019; 199: 784-794.

2 Hong J, Lee SH, Ryu BH, et al. Diagnostic usefulness of bronchoalveolar lavage fluid xpert MTB/RIF in pauci-bacillary pulmonary tuberculosis. Infect Dis (Lond) 2018; 50: 725-727.

3 Jin CE, Koo B, Lee EY, et al. Simple and label-free pathogen enrichment via homobifunctional imidoesters using a microfluidic (SLIM) system for ultrasensitive pathogen detection in various clinical specimens. Biosens Bioelectron 2018; 111: 66-73.

4 Park JH, Jin CE, Koo B, et al. A simple microfluidic assay for diagnosing tuberculous meningitis in HIV-uninfected patients. J Clin Microbiol 2019; 57: e01975-18.

5 Dorman SE, Schumacher SG, Alland D, et al. Xpert MTB/RIF Ultra for detection of Mycobacterium tuberculosis and rifampicin resistance: a prospective multicentre diagnostic accuracy study. Lancet Infect Dis 2018; 18: 76-84.

$6 \quad$ Pai M, Behr MA, Dowdy D, et al. Tuberculosis. Nat Rev Dis Primers 2016; 2: 16076.

7 Drain PK, Bajema KL, Dowdy D, et al. Incipient and subclinical tuberculosis: a clinical review of early stages and progression of infection. Clin Microbiol Rev 2018; 31: e00021-18.

8 Kim SH, Cho OH, Park SJ, et al. Rapid diagnosis of tuberculous meningitis by T cell-based assays on peripheral blood and cerebrospinal fluid mononuclear cells. Clin Infect Dis 2010; 50: 1349-1358.

9 Kim SH, Choi SJ, Kim HB, et al. Diagnostic usefulness of a T-cell based assay for extrapulmonary tuberculosis. Arch Intern Med 2007; 167: 2255-2259.

10 Kim JY, Park JH, Kim MC, et al. Combined IFN-gamma and TNF-alpha releasing assay for differentiating active tuberculosis from latent tuberculosis infection. J Infect 2018; 77: 314-320. 